\title{
Independence of physical activity and screen time as predictors of cardiorespiratory fitness in youth
}

\author{
Gavin R.H. Sandercock ${ }^{1}$ and Ayodele A. Ogunleye ${ }^{1}$
}

BACKGROUND: Screen time (ST) is associated with physical activity (PA) levels. We aimed to investigate the relationship between ST and fitness.

METHODS: We assessed fitness and daily ST in 7,466 10-16$y$-old schoolchildren from the east of England. The differences in fitness between ST groups, and the association between ST and fitness were assessed.

RESULTS: ST was negatively associated with fitness in boys of all ages $(P<0.001)$ and girls in grades $6-9(P<0.005)$. Boys were less likely to be fit if they reported $>2 \mathrm{~h} / \mathrm{d}$ ST (odds ratio (OR): $0.70,95 \%$ confidence interval (Cl): $0.58-0.85$ ) or $>4 \mathrm{~h} / \mathrm{d}$ (OR: $0.45,95 \% \mathrm{Cl}: 0.35-0.57$ ) ST, as were girls reporting $>4 \mathrm{~h} / \mathrm{d}$ ST (OR: 0.58, 95\% Cl: 0.43-0.78). Controlling for PA levels attenuated these odds in boys reporting $>2 \mathrm{~h} / \mathrm{d}$ ST (OR: $0.81,95 \% \mathrm{Cl}$ : $0.66-0.91$ ) or $>4$ h/d ST (OR: $0.65,95 \%$ Cl: $0.50-0.84$ ) and in girls reporting >4 h/d ST (OR: $0.68,95 \% \mathrm{Cl}: 0.50-0.93$ ).

CONCLUSION: These first data from English children show a negative association between ST and fitness in youth. Of note, very high levels of daily ST ( $>4 \mathrm{~h}$ ) are associated with poor fitness. Some of the associations were mediated by PA levels. Our data support international recommendations to limit ST to $<2 \mathrm{~h} / \mathrm{d}$; we believe such guidance should be issued in the United Kingdom.

$\mathbf{T}$ ime spent in sedentary behavior is independently linked to poor metabolic health in adults and children $(1,2)$. Although there are many sedentary behaviors, small-screen media engagement (screen time, ST) remains the most prevalent (3) and is a commonly used indicator of overall levels of sedentariness in large-scale epidemiological studies $(2,4-6)$.

The moderate negative cross-sectional relationship between ST and physical activity (PA) is well established and would seem intuitive $(6,7)$. Although ST and PA are clearly interrelated (8), a reciprocal relationship between them cannot be assumed (high ST is not necessarily associated with lower levels of PA). Multivariate analyses have shown that they independently predict poor metabolic health $(1,9)$. This has led to the suggestion they should be treated as separate entities or constructs (9).

Cardiorespiratory fitness (herein fitness) is an outcome of PA and provides a useful objective end point by which the possible impact of excessive sedentariness in youth can be measured (10). Fitness is easier to measure than PA, tracks more strongly through childhood (11), and is better related to health outcomes in children (12) and adults (13). Studies examining the relationship between television viewing and fitness have shown no significant relationship (14-18) or inconsistent findings (18). Most were limited by small samples sizes and in their measurement only of television viewing, which poorly reflects overall sedentary behavior (19). A recent large study using a composite ST measure (television viewing, computer use, games console use) and cardiorespiratory fitness found a small, negative association between ST and cardiorespiratory fitness in boys but not girls (10). There were also differences in the relationship across the three age categories. Although the sample was large and representative, fitness was expressed only as a continuous variable (laps completed on the 20-m shuttlerun test). In population research, fitness may be better represented as a categorical variable score that is related to a criterion-referenced standard indicative of health-related fitness.

The aims of the present study were to verify findings of previous studies using a large sample of English youth. Second, we aimed to examine the relationship between ST and cardiorespiratory fitness expressed as a criterion-referenced standard related to health. Finally, we sought to explore the role PA plays in mediating the relationship between ST and fitness.

\section{RESULTS}

Descriptive characteristics are shown in Table 1. The prevalence of ST $(>2 \mathrm{~h} / \mathrm{d})$ was $35.7 \%$; low ST $(<2 \mathrm{~h} / \mathrm{d})$ was more common in girls whereas very high ST $(>4 \mathrm{~h} / \mathrm{d})$ was significantly more prevalent in boys. Overall levels of fitness expressed as shuttles completed and peak oxygen uptake were higher in boys. Prevalence of unfitness was also higher in boys as a result of the higher cutoff used to define health-related fitness (20).

Figure 1 shows the results of the one-way ANOVA comparing the number of shuttles run between ST groups. In boys, there was a significant $(P<0.001)$ main effect for ST in every age category. Post hoc analyses showed significant differences in the number of shuttles completed between all three ST groups in each age group. In girls, there were significant main effects in grades $7-8$ and $9-10$ but not in grades $10-11(F=1.89$, $P=0.155)$. Post hoc analyses showed grade $7-8$ girls reporting very high ST completed significantly fewer shuttles than those who reported low ST. In grade 6-7 girls, significant differences 
Table 1. Descriptive characteristics of the sample according to sex

\begin{tabular}{|c|c|c|c|c|c|c|}
\hline & \multicolumn{2}{|c|}{ All } & \multicolumn{2}{|c|}{ Boys } & \multicolumn{2}{|c|}{ Girls } \\
\hline & $\begin{array}{c}\text { Mean } \\
\text { or } \%\end{array}$ & $\begin{array}{l}\text { SD } \\
\text { or } n\end{array}$ & $\begin{array}{c}\text { Mean } \\
\text { or } \%\end{array}$ & $\begin{array}{l}\text { SD } \\
\text { or } n\end{array}$ & $\begin{array}{c}\text { Mean } \\
\text { or } \%\end{array}$ & $\begin{array}{l}\text { SD } \\
\text { or } n\end{array}$ \\
\hline Number & 100 & 7,466 & 53 & 3,966 & 47 & 3,500 \\
\hline Age (years) & 13.0 & 1.4 & 13.1 & 1.5 & 13.0 & 1.5 \\
\hline $\operatorname{BMI}\left(\mathrm{kg} / \mathrm{m}^{2}\right)$ & 20.3 & 3.6 & 20.2 & 3.6 & 20.4 & 3.6 \\
\hline BMI z-score & 0.50 & 1.1 & 0.58 & 1.1 & 0.51 & $1.1^{*}$ \\
\hline PACER shuttles & 43.3 & 23 & 51.0 & 25 & 34.1 & $15.1^{*}$ \\
\hline $\mathrm{V}_{\text {2peak }}(\mathrm{ml} / \mathrm{kg} / \mathrm{min})$ & 43.6 & 6.0 & 45.7 & 6.2 & 41.3 & $4.9^{*}$ \\
\hline Unfit (\%) & 23.0 & 1,572 & 26.7 & 969 & 18.8 & $603^{* *}$ \\
\hline Screen time $<2 \mathrm{~h}$ & 64.3 & 4,839 & 62.0 & 2,475 & 66.9 & $2,352^{* *}$ \\
\hline Screen time $2-4 \mathrm{~h}$ & 23.9 & 1,797 & 24.6 & 981 & 23.2 & 816 \\
\hline Screen time $>4 \mathrm{~h}$ & 11.8 & 886 & 13.4 & 535 & 9.9 & $349^{* *}$ \\
\hline
\end{tabular}

BMI z-score calculated (ref. 34); $V_{2}$ enek estimated using Leger's equation (ref. 36). Unfit determined as score below the Fitnessgram standards (ref. 20).

$\mathrm{Vo}_{2 \text { peak }}$ 'peak oxygen uptake

${ }^{*} P<0.05 ;{ }^{*} P<0.01$

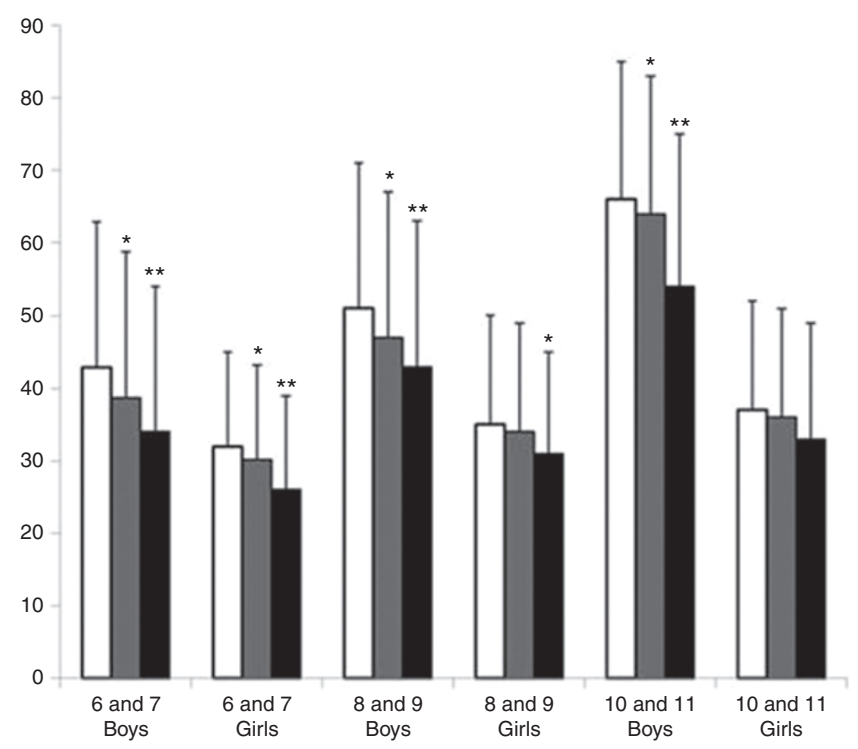

Figure 1. Median (interquartile range) differences in PACER performance (shuttles completed) according to screen time group: sample divided by grade and sex. White bars, low screen time group $(<2 \mathrm{~h} / \mathrm{d})$; gray bars, high screen time group ( $2-4 \mathrm{~h} / \mathrm{d})$; black bars, very high screen time group $(>4 \mathrm{~h} / \mathrm{d})$. Mean (SD) age (years) grades 6 and 7: 11.4 (0.6); grades 7 and 8: 13.0 (0.6); and grades 10 and $11: 14.8(0.6) .{ }^{*} P<0.05$, significantly less fit than low screen time group. ${ }^{* *} P<0.05$, significantly less fit than high screen time group.

in fitness between all ST groups were observed. There was a dose-response relationship between the three ST categories and binary classification of fitness. Trend analysis revealed a sequentially higher proportions of unfit schoolchildren in ascending ST groups $\left(\chi_{\text {trend }}^{2}=78.90\right.$, degrees of freedom $\left.=1, P<0.001\right)$.

\section{Binary Logistic Regression}

When the entire data set was analyzed controlling for age, sex, deprivation, ethnicity, and BMI, the likelihood of being fit was significantly lower in participants reporting high (odds ratio, OR: $0.66,95 \%$ confidence interval (CI): 0.50-0.78) and very high (OR: $0.48,95 \% \mathrm{CI}: 0.40-0.58)$ ST than in those who
Table 2. Binary logistic regression analysis to predict likelihood of being classified as fit

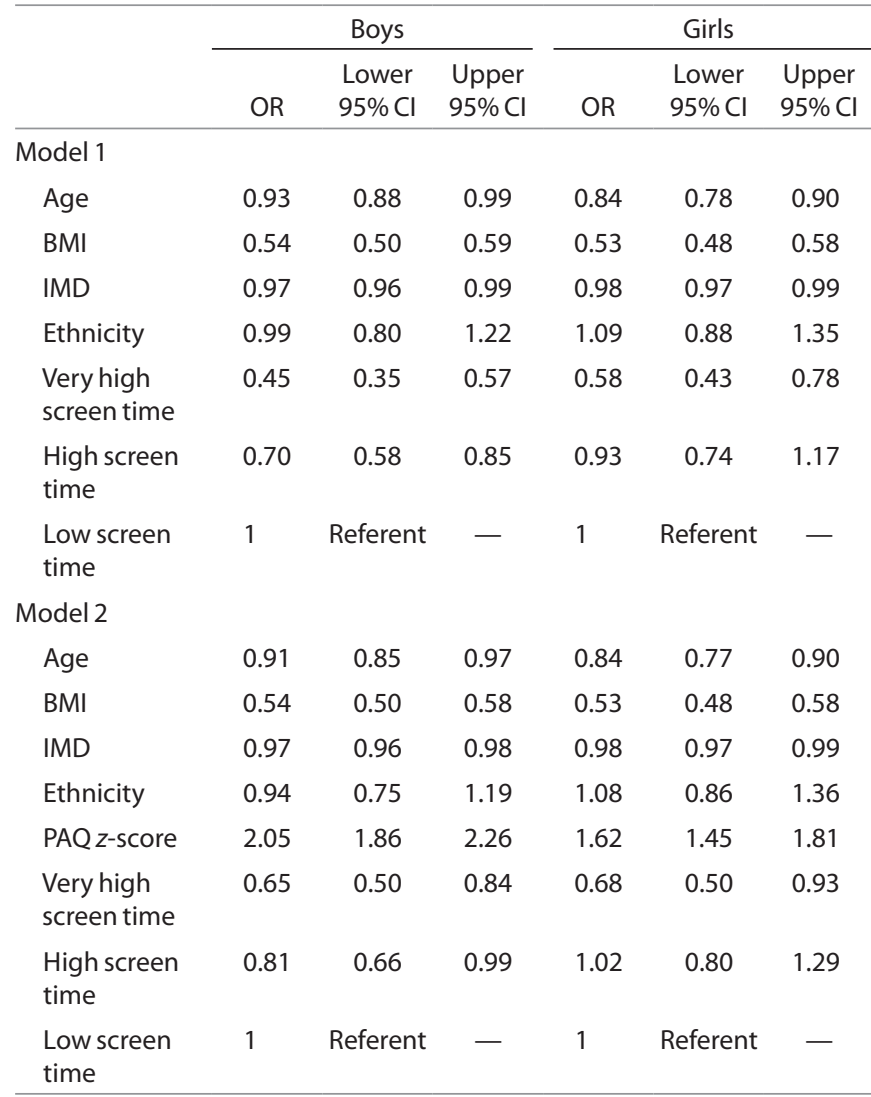

Deprivation measured using English Indices of Multiple Deprivations (ref. 35); PAQ score (ref. 32); BMI $z$-score (ref. 34). Fitness defined as exceeding the Fitnessgram Lower Healthy Fitness Zone Cut-Off. Low, $<2$ h; high, 2-4h; very high, $>4$ h daily screen time.

$\mathrm{Cl}$, confidence interval; IMD, index of multiple deprivation; $\mathrm{OR}$, odds ratio; $\mathrm{PAQ}$, physical activity questionnaire.

reported low levels. Sex was a significant, independent predictor of fitness, with boys less likely to be classified as fit (OR: 0.35, 95\% CI: 0.31-0.42). Further analyses were performed in boys and girls separately.

Results of the two logistic regression models are shown in Table 2. As compared with those with low ST, boys reporting high or very high ST were less likely to meet criterion-referenced fitness standards. Of note, boys reporting very high ST were less than half as likely to be fit as compared with those in the low ST group (OR: 0.45, 95\% CI: 0.35-0.57). Controlling for PA (physical activity questionnaire (PAQ) $z$-score) slightly reduced the odds of being unfit, but this remained significant in those boys with high (OR: $0.81,95 \%$ CI: $0.66-0.91$ ) and those with very high (OR: $0.65,95 \%$ CI: $0.50-0.84$ ) ST.

The relationship between ST and fitness was less marked in girls, and there was no significant difference in the likelihood of being fit between low and high ST groups. Girls who reported very high ST were, however, less likely to be fit than those in the low ST group (OR: 0.58, 95\% CI: 0.43-0.78). These odds were slightly reduced but remained significant after controlling for PA levels (OR: 0.68, 95\% CI: 0.50-0.93).

The total effect $(c)$ and direct effect $\left(c^{\prime}\right)$ of ST on fitness were $-0.056(P<0.001)$ and $-0.028(P<0.001)$, respectively. 


\section{Articles | Sandercock and Ogunleye}

On the basis of 5,000 bootstrap samples, the total indirect effect of ST on fitness was $-0.029,95 \%$ CI: -0.034 to -0.024 , that was OR: 0.97, bias-corrected and accelerated 95\% CI: $0.97-0.98$, which leads to a rejection of the hypothesis that the total indirect effect was zero $(P<0.001)$. These estimates are similar to that of the normal theory tests for indirect effects. Because the assumption of normality of the sampling distribution of indirect effects was arguable, we bootstrapped the indirect effects of fitness on ST. In agreement with results of the product-of-coefficients strategy, PAQ $z$-score was an important mediator of the fitness and ST relationship. On the basis of the $95 \% \mathrm{CI}$, we concluded that the indirect effect was indeed significantly different from zero $(P<0.001)$, implying that PA levels partially mediate the association between ST and fitness.

\section{DISCUSSION}

Sedentary behavior and PA are separate constructs (21) that can independently predict metabolic risk in children $(1,2,5)$. As a marker of sedentary behavior, ST shows consistent but weak associations with higher adiposity (22) and is moderately associated with lower PA in children (23). The association between ST and fitness is less studied, and findings have been mixed. This study aimed to assess the relationship between ST and fitness expressed as a health-related criterion standard (20) in a large sample of children and adolescents.

The present data show a significant inverse relationship between ST and cardiorespiratory fitness in children of both sexes across a range of ages (10-16y). When fitness was expressed as a continuous, test performance score, our data partly agree with those reported previously in Australian schoolchildren (10) because we also found significantly lower fitness scores in boys reporting high ST. Our use of three ST categories provides further information by showing a "dose-response" type relationship between ST and fitness in boys. Hardy et al. (10) found no difference in girls' fitness according to ST, whereas we found significantly lower fitness only in our additional category of very high ST. These are the first data to show decrements in fitness in accordance with ST in a dose-response manner. From a public health perspective, these data suggest that very high ST is associated with lower fitness in boys and girls and hence should be avoided. The data also support some potential health benefits of current recommendations $(24,25)$ to limit daily ST to $<2 \mathrm{~h}$.

The high ST group represents children previously described as "heavy users" (2). Eisenmann et al. (2) found higher metabolic risk scores in heavy users as compared with children reporting either low $(<2 \mathrm{~h} / \mathrm{d})$ or higher $(2-4 \mathrm{~h} / \mathrm{d}) \mathrm{ST}$. Schoolchildren who report very high daily ST are less physically active at school and even during physical education lessons, as well as at the more intuitive times such as after school and weekends (4). The current data suggest that such a lifestyle, involving very high ST and habitually low PA, is associated with a significantly lower likelihood of achieving health-related levels of cardiorespiratory fitness.

Such findings are at odds with studies showing no relationship between ST and fitness in youth. These earlier studies were often limited by measurement of television viewing only, by small sample size, and by the use of arbitrary cutoffs for ST categorization (14-18). Only Hardy et al. (10) used categories of ST based on current guideline daily amounts (low: $<2 \mathrm{~h} / \mathrm{d}$ vs. high: $>2 \mathrm{~h} / \mathrm{d}$ ) for children and adolescents (24). In the current study, ST was more strongly associated with fitness in boys than in girls, and the present data suggest that perhaps only very high ST is associated with lower fitness in girls, a notion that has not been addressed in any previous research.

Fitness may have several advantages over PA as an indicator of health. Fitness can be objectively measured in large samples with relative ease using validated field tests capable of producing nearmaximal effort in children and adolescents (26). More important, fitness is better related to disease status in adults than PA $(12,13)$. Finally, scores on fitness tests can be expressed as criterion-referenced health standards indicative of future or present disease risk in children. A number of cut-points are available (27), all of which tend to be quite similar. We used the Fitnessgram Lower Healthy Fitness Zone cutoffs (20) because these are linked to predictions of future ill-health (28) and to allow comparisons of our findings with recent research (10).

\section{Predictors of Criterion-Referenced Fitness}

Even when controlling for several potential confounding variables, ST remained a significant independent predictor of fitness in boys and girls. There was a clear dose-response relationship in boys, in whom very high ST was associated with a very low likelihood of being classified as fit. In fact, the very high ST group's odds of being fit were less than half those of those in the low ST group. In girls, the association was not as strong, and there was less evidence of a dose-response. Girls with high ST were no less likely to be fit than those reporting lower levels. The likelihood of being fit was, however, markedly (42\%) lower in high vs. low ST groups (OR: 0.58, 95\% CI: 0.43-0.78).

These data support findings from studies that have expressed fitness as a continuous variable in which a larger effect size for between-group difference is evident in boys than in girls (10). It seems that boys report higher levels of ST and that these may be more closely associated with fitness in boys than they are in girls. These patterns in fitness are analogous to trends in PA that we have previously reported in this population showing larger effect sizes for differences in PA according to ST in boys than girls (4). No study has previously attempted to determine whether associations between ST and fitness are independent of PA. Although lower PA, displaced by ST, appears the most intuitive mechanism to link high ST to low fitness, previous research has shown that TV viewing habits can change independently of PA habits (21). The independence of (high) ST and (low) PA from one another was demonstrated previously by Ekelund et al. (9), who showed them to be independent predictors of elevated metabolic risk scores in children.

\section{Independence of PA and ST as Predictors of Fitness}

ST, PA, and fitness are all interrelated, therefore, we hypothesized that controlling for PA would eliminate the relationship between ST and fitness. Given that fitness is influenced by habitual PA levels, we sought to test the hypothesis that higher 
PA in low ST groups appears the most plausible explanation for the association of ST with fitness.

In boys, controlling for PA attenuated the odds of being fit according to ST, but they remained significantly lower in the high (OR: $0.81,95 \%$ CI: $0.66-0.99$ ) and particularly in the very high (OR: 0.65, 95\% CI: 0.50-0.84) ST groups. Similarly, girls with very high ST were less likely to be fit even after controlling for PA (OR: 0.68, 95\% CI: 0.50-0.93). This suggests that boys exceeding $2 \mathrm{~h}$ and girls exceeding $4 \mathrm{~h}$ daily ST are more likely to have low cardiorespiratory fitness independent of their PA levels. This is a novel and important finding and supports the findings of inactive physiology studies showing such relationships in adults (29). Both fitness and PA levels are associated with ST.

Furthermore, our mediation analysis, using PA as a mediator of the association between fitness and ST, shows that PA levels explain, in part, the difference in fitness observed across ST categories. A partial mediation was observed in the relationship between fitness and ST after controlling for the mediating variable, PA. The directions of the $\alpha$ and $\beta$ paths (Figure 1) suggest that decreasing ST may lead to increase in PA levels, which in turn leads to greater fitness. However, this result should be treated with caution because increasing PA may reduce the negative association between fitness and ST. Therefore, intervention targeting PA levels may decrease ST in youth who are unfit.

These data, like those of Eisenmann et al. (2), suggest that very high ST $(>4 \mathrm{~h} / \mathrm{d})$ may be an important threshold for children's health. These data also support the notion that PA and ST independently contribute to poor health outcomes in children (9).

It has long been accepted that adequate levels of PA promote good health, whereas the potential negative impact of sedentary behaviors is a relatively recent idea $(19,29)$. The intuitive pathway by which sedentary activities such as ST could be associated with low fitness is by direct displacement of vigorous active pursuits that are needed to potentiate increases in fitness. But there is an increasing body of evidence to show that time spent sitting is predictive of poor health in adults independent of PA (29). The present data are the first to demonstrate a relationship between ST and health-related measures of physical fitness in children. Our findings strongly suggest that ST should be kept below $4 \mathrm{~h} / \mathrm{d}$ because of this independent, negative association with cardiorespiratory fitness.

Cardiorespiratory fitness can be thought of as a health outcome in children representing typical lifestyle (PA and body weight levels), whereas PA is a behavior an individual can quickly and transiently modify. Perhaps for this reason, or because it can be objectively measured, fitness is a better predictor of health outcomes than is PA $(12,13)$. In young people, more prone to recall bias, objective measures of fitness have clear advantages. It is in adulthood, however, that low PA or fitness is likely to manifest as diseases (28). Although physical activity tracks very poorly, cardiorespiratory fitness tracks moderately from childhood to adulthood (30). This may also suggest that fitness is a more appropriate measure for health surveillance.
The associations seen throughout this study were stronger in boys than girls. There is evidence that girls spend more time in sedentary but non-screen-based activities such as talking on the phone and "hanging out" (19,31). ST may, therefore, simply be a better index of sedentariness in boys than girls, but other explanations are also possible. Our ST measure cannot determine the relative distribution of ST between, for instance, computer use and television viewing. Greater television viewing, accompanied by unhealthful snacking behaviors, may have an effect on body composition (BMI is higher in boys), which could influence fitness measured using our weightbearing test. It is also possible that the independence of ST from PA as a predictor of fitness may be due to methodological reasons. Our measure of fitness was objective, used established methods, and was expressed as criterion-referenced score. Unfortunately, this was not possible to achieve with our measure of PA. Although the PAQ is both reliable and valid (32), answers are still prone to either poor recall or recall bias. The PAQ also does not measure the intensity of exercise and activities that each child reports but provides instead descriptive, quantitative estimates of the variety of activities undertaken, frequency of certain activities, level of effort in physical education classes, and overall activity levels for the past $7 \mathrm{~d}$. Repeated bouts of high-intensity activity are needed to promote increases in cardiorespiratory fitness, but these are difficult to determine from the PAQ.

Our study has several other limitations. Our cross-sectional design means that we cannot make inferences regarding the direction of cause and effect between ST and fitness, and longitudinal data are needed to address this. Although we made a valid and objective measurement of fitness, we relied on selfreport to gain our estimates of PA and ST. We have found good validity and reliability of our ST measure (23), but it cannot account for differences between weekend and weekday ST. We do not claim to have made an accurate assessment of the total sedentary time of our sample, but ST remains the predominant sedentary behavior (3) and it should be regarded as a proxy of total sedentary time useful for within-subject comparisons in large studies such as this one.

\section{Conclusion}

Despite these limitations, the current study provides original findings regarding the relationship between ST and fitness in children. These are the first data on English children, a group that typically has levels of television viewing and household games console and personal computer ownership comparable with other developed countries including the United States, Canada, and Australia $(8,10,22)$. This is the first study to show a significant negative relationship between ST and cardiorespiratory fitness expressed as a health-related criterion standard. This likelihood of being unfit was much greater in boys who exceed current international recommendations $(24,25)$ for daily ST and in girls with very high levels. This relationship was independent of age, deprivation, ethnicity, and BMI. Most important, our second analysis demonstrates that, in children with very high ST, the likelihood of being unfit was 


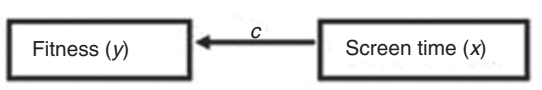

b

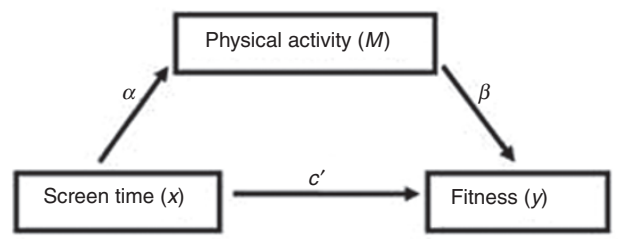

Figure 2. Proposed mediation pathways. (a) Direct effect (pathway c), not including mediator. (b) Illustrating adjusted direct (pathway $c^{\prime}$ ) and indirect effects (pathways $\alpha$ and $\beta$ ) through potential mediation by physical activity levels.

even independent of PA levels. These data suggest that existing guidance to limit $\mathrm{ST}$ to $<2 \mathrm{~h} / \mathrm{d}$ is adequate. The data cannot, however, confirm whether this cutoff is optimal because of the lack of additional categories below $2 \mathrm{~h} / \mathrm{d}$, and further research is warranted in this area. We found a dose-response relationship between ST and fitness that was only partially mediated by differences in PA. Our mediation analysis suggests that ST is independently associated with fitness via as yet unknown mechanisms unrelated to PA levels. These mechanisms require elucidation. Regardless of individual PA levels, ST should definitely be kept below 4 , and preferably below $2 \mathrm{~h} / \mathrm{d}$ if children are to be able to achieve health-related fitness standards.

\section{METHODS}

\section{Participants and Sample}

Participants were part of the ongoing East of England Healthy Hearts Study; details of the sample and methodology are available elsewhere (33). Briefly, the study is an ongoing survey that was approved by the University of Essex Ethical Review Board. Informed consent was gained from the schools, parents, and from each child at point of contact. All schools were state schools in the east of England. Pupils normally attending physical education were potentially included in the study; exclusion criteria were the presence of known illness (such as underlying cardiomyopathy) and lack of parental or pupil consent. Schools provided consent for pupils to be tested, and we used an additional opt-out approach to parental consent. Finally, verbal consent was required from each participant at the point of testing. This approach resulted in response rate of $98.2 \%$.

A total of 7,466 schoolchildren between 10 and 16 y (53\% boys) were involved in this cross-sectional study. Children were weighed and their weight and height were recorded to the nearest $0.1 \mathrm{~kg}$ and $0.1 \mathrm{~cm}$, respectively. Stature was measured using Seca Leicester Height Measure (Seca \& Co. KG., Hamburg, Germany) and mass using a Seca 888 digital scale (Seca \& Co. KG.). We ensured all participants were wearing light clothing (T-shirts and shorts) and without shoes. BMI was calculated from the mass and height and expressed as an age- and sex-normalized $z$-score (34).

Daily ST was assessed using our own, validated self-report questionnaire (4). ST responses were categorized in two ways. The response categories: "none," "30 min or less," "30 min-1 h," " $1-2$ h," " $2-4$ h," or " $>4$ h," were condensed into three categories: "low" $(<2 \mathrm{~h})$, "high" $(2-4 \mathrm{~h})$ based on international guidance on limiting pediatric ST, and finally, "very high" (>4h), a category used previously to describe heavy use $(2,4)$.

Participants also self-reported their ethnicity via menu-based responses taken from the UK National Census as well as their home postcode, from which we determined the area-level deprivation index using national census data (35). The current study used the English Index of Multiple Deprivation. This has been discussed before (23), but briefly the English Index of Multiple Deprivation is a more detailed measure than simple or proxy estimates of socioeconomic status based on education or employment given that it covers many aspects of neighborhood-level deprivation. The measure has 37 indicators in seven domains: income, living environment, education, employment, housing, health, and crime levels.

Participants then completed the Fitnessgram PACER test to estimate their cardiorespiratory fitness as described previously (33). Participants had previously taken part in the 20 -m shuttle-run test as part of their physical education. Participants were reminded by both the instructions on the PACER CD and a researcher to "run for as long as possible." The test requires volunteers to run back and forth over a marked distance of $20 \mathrm{~m}$ in time with an audible signal. The test starts at an initial running speed of $8.0 \mathrm{~km} / \mathrm{h}$ and increases initially by $1 \mathrm{~km} / \mathrm{h}$ after $2 \mathrm{~min}$ then by $0.5 \mathrm{~km} / \mathrm{h}$ each minute thereafter. Researchers acted as "spotters" and recorded the final shuttle count at either the point of volitional exhaustion or when the participant failed to maintain the required running speed twice. For comparison with previous data, we expressed fitness as the total number of shuttles completed. As a descriptive characteristic, we also converted this to peak oxygen consumption (36). Total shuttles completed is dependent on participant age, so we grouped participants into three age categories for this analysis as others have (10). We also expressed fitness as a binary variable using the lower Fitnessgram PACER Healthy Fitness Zone cutoffs (20). If the participants' total completed shuttle count was above their age- and sex-specific cutoff, they were classed as "fit"; otherwise, participants were classed as "unfit."

Each participant completed the PAQ for adolescents (PAQ-A) or children (PAQ-C). Participants of high school age ( $>11$ y) completed the PAQ-A and primary schoolchildren $(<11$ y) completed the PAQ-C. The PAQ-A or PAQ-C, which contains different elements of PA, was used to determine participants' PA levels; this we have detailed elsewhere (23). This instrument has been previously validated $(32,37)$. Age- and sex-standardized PAQ scores ( $z$-scores) were generated from participant's overall PAQ score.

\section{Data Analysis}

Differences in boys and girls were assessed by independent $t$-tests or $\chi^{2}$ analysis as appropriate. We divided participants into three categories based on school grades (grades: 6-7, 8-9, and 10-11). The $\chi^{2}$ test for trend $\left(\chi_{\text {trend }}^{2}\right.$, degrees of freedom, $P$ value) was used for a dose-response model and was reported for differences in distribution of ST with ordered categories. ANOVA was performed in boys and girls within each group separately. Post hoc (Tukey) tests were used to determine the differences in PACER performance between ST groups. Analysis was performed on the natural logarithm of laps completed to ensure normality of distribution (10). We then performed binary logistic regression analyses on the whole data set to determine the likelihood of each participant being classified as fit according to his or her ST. The initial analysis controlled for age, sex, deprivation, ethnicity, and BMI. We then split the data by sex and repeated this analysis. A second model also included self-reported PA as a covariate to examine whether associations between fitness and ST were dependent on PA.

A mediation variable analysis was conducted using the bootstrapbased product-of-coefficient test with fitness as the outcome variable. A set of generalized linear models were fitted to evaluate the contribution of the mediating variable, which is PA in this case (Figure 2). Models were generated using a more robust current practice to generate $\alpha, \beta, c$, and $c^{\prime}$ (38). We estimated the total effect of ST on fitness ( $c$ coefficient); in addition to this, we estimated the association between ST, independent variable, and the potential mediator, PA, ( $\alpha$ coefficient); the independent effects of the potential mediator, PA, on the outcome, fitness, ( $\beta$ coefficients) using a generalized linear modeling analyses, i.e., regressing fitness and mediating variable PAQ $z$-scores. Step 3: estimating the association between outcome (fitness) and independent variable (PA) ( $c^{\prime}$ coefficient) and computing the product of the two coefficients $\alpha \beta$ (product-of-coefficient estimate of a mediated effect), representing the mediated effect, for each bootstrap sample; and step 4: computing the bootstrap bias-corrected 95\% CIs around the bootstrap estimates of $\alpha \beta$. Product-of-coefficient estimate is recommended with logistic regression, where our outcome variable (fitness) is categorical (39). In the mediation analysis, ST represents our independent 
variable, which ranges from 0 to 5 depending on participant's response as explained above. The mediation analysis was run as a script, written by Preacher and Hayes (40) on SPSS (IBM, Armonk, NY), using 5,000 bootstrap resamples to determine whether the indirect pathway through PA was significantly different from 0 . This was done because bootstrapping is considered to be more robust. Bias-corrected and accelerated 95\% CIs were produced for the sizes of the indirect effects. We calculated OR and its associated $95 \%$ CI by taking the antilogarithm (exponential) of regression coefficients and antilogarithm of $95 \%$ biascorrected and accelerated CI, respectively. All analyses were performed using SPSS software, version 19.

\section{ACKNOWLEDGMENTS}

We acknowledge all the people who contributed to the development of this project, especially those who were involved in the data collection process in the schools. We also thank all of the children and the physical education department staff of the schools who participated in this project.

\section{STATEMENT OF FINANCIAL SUPPORT}

This study was funded by the University of Essex Research Promotion Fund (grant DB014B5).

Disclosure: The authors declared no conflict of interest.

\section{REFERENCES}

1. Mark AE, Janssen I. Relationship between screen time and metabolic syndrome in adolescents. J Public Health (Oxf) 2008;30:153-60.

2. Eisenmann JC, Bartee RT, Wang MQ. Physical activity, TV viewing, and weight in U.S. youth: 1999 Youth Risk Behavior Survey. Obes Res 2002;10:379-85.

3. Biddle SJ, Gorely T, Marshall SJ, Cameron N. The prevalence of sedentary behavior and physical activity in leisure time: A study of Scottish adolescents using ecological momentary assessment. Prev Med 2009;48:151-5.

4. Sandercock GRH, Ogunleye A, Voss C. Screen time and physical activity in youth: thief of time or lifestyle choice? J Phys Act Health 2012;9:977-84

5. Steele RM, van Sluijs EM, Cassidy A, Griffin SJ, Ekelund U. Targeting sedentary time or moderate- and vigorous-intensity activity: independent relations with adiposity in a population-based sample of 10-y-old British children. Am J Clin Nutr 2009;90:1185-92.

6. Vandewater EA, Bickham DS, Lee JH. Time well spent? Relating television use to children's free-time activities. Pediatrics 2006;117:e181-91.

7. DuRant RH, Baranowski T, Johnson M, Thompson WO. The relationship among television watching, physical activity, and body composition of young children. Pediatrics 1994;94(4 Pt 1):449-55.

8. Marshall SJ, Gorely T, Biddle SJ. A descriptive epidemiology of screenbased media use in youth: a review and critique. J Adolesc 2006;29:333-49.

9. Ekelund U, Brage S, Froberg K, et al. TV viewing and physical activity are independently associated with metabolic risk in children: the European Youth Heart Study. PLoS Med 2006;3:e488.

10. Hardy LL, Dobbins TA, Denney-Wilson EA, Okely AD, Booth ML. Sedentariness, small-screen recreation, and fitness in youth. Am J Prev Med 2009;36:120-5.

11. Andersen LB, Haraldsdóttir J. Tracking of cardiovascular disease risk factors including maximal oxygen uptake and physical activity from late teenage to adulthood. An 8-year follow-up study. J Intern Med 1993;234:309-15.

12. Hurtig-Wennlöf A, Ruiz JR, Harro M, Sjöström M. Cardiorespiratory fitness relates more strongly than physical activity to cardiovascular disease risk factors in healthy children and adolescents: the European Youth Heart Study. Eur J Cardiovasc Prev Rehabil 2007;14:575-81.

13. Williams PT. Physical fitness and activity as separate heart disease risk factors: a meta-analysis. Med Sci Sports Exerc 2001;33:754-61.

14. Tucker LA. The relationship of television viewing to physical fitness and obesity. Adolescence 1986;21:797-806.

15. Katzmarzyk PT, Malina RM, Song TM, Bouchard C. Television viewing, physical activity, and health-related fitness of youth in the Québec Family Study. J Adolesc Health 1998;23:318-25.

16. Armstrong CA, Sallis JF, Alcaraz JE, Kolody B, McKenzie TL, Hovell MF. Children's television viewing, body fat, and physical fitness. Am J Health Promot 1998;12:363-8.
17. Kerner M, Kurrant A, Kalinski MI. Leisure-time physical activity, sedentary behavior, and fitness of high school girls. Eur J Sports Science 2004;4:1-17.

18. Grund A, Krause H, Siewers M, Rieckert H, Müller MJ. Is TV viewing an index of physical activity and fitness in overweight and normal weight children? Public Health Nutr 2001;4:1245-51.

19. Biddle SJ, Gorely T, Marshall SJ. Is television viewing a suitable marker of sedentary behavior in young people? Ann Behav Med 2009;38:147-53.

20. Meredith MD, Welk GJ. Chapter 9. Interpreting FITNESSGRAM results. In Meredith MD, Welk GJ (eds). Fitnessgram/Activitygram Administration Manual. Champaign, IL: Human Kinetics, 2007:59-68.

21. Taveras EM, Field AE, Berkey CS, et al. Longitudinal relationship between television viewing and leisure-time physical activity during adolescence. Pediatrics 2007;119:e314-9.

22. Marshall SJ, Biddle SJ, Gorely T, Cameron N, Murdey I. Relationships between media use, body fatness and physical activity in children and youth: a meta-analysis. Int J Obes Relat Metab Disord 2004;28:1238-46.

23. Ogunleye AA, Voss C, Sandercock GR. Prevalence of high screen time in English youth: association with deprivation and physical activity. J Public Health (Oxf) 2012;34:46-53.

24. Barlow SE; Expert Committee. Expert committee recommendations regarding the prevention, assessment, and treatment of child and adolescent overweight and obesity: summary report. Pediatrics 2007;120:Suppl 4:S164-92.

25. Tremblay MS, Leblanc AG, Janssen I, et al. Canadian sedentary behaviour guidelines for children and youth. Appl Physiol Nutr Metab 2011;36: 59-64; 65-71.

26. Voss C, Sandercock G. Does the twenty meter shuttle-run test elicit maximal effort in 11- to 16-year-olds? Pediatr Exerc Sci 2009;21:55-62.

27. Sandercock G, Voss C, Cohen D, Taylor M, Stasinopoulos DM. Centile curves and normative values for the twenty metre shuttle-run test in English schoolchildren. J Sports Sci 2012;30:679-87.

28. Blair SN, Kohl HW 3rd, Paffenbarger RS Jr, Clark DG, Cooper KH, Gibbons LW. Physical fitness and all-cause mortality. A prospective study of healthy men and women. JAMA 1989;262:2395-401.

29. Hamilton MT, Healy GN, Dunstan DW, Zderic TW, Owen N. Too Little Exercise and Too Much Sitting: Inactivity Physiology and the Need for New Recommendations on Sedentary Behavior. Curr Cardiovasc Risk Rep 2008;2:292-8.

30. Andersen LB, Hasselstrøm H, Grønfeldt V, Hansen SE, Karsten F. The relationship between physical fitness and clustered risk, and tracking of clustered risk from adolescence to young adulthood: eight years follow-up in the Danish Youth and Sport Study. Int J Behav Nutr Phys Act 2004;1:6.

31. Biddle SJ, Marshall SJ, Gorely T, Cameron N. Temporal and environmental patterns of sedentary and active behaviors during adolescents' leisure time. Int J Behav Med 2009;16:278-86.

32. Kowalski KC, Crocker PRE, Kowalski NP. Convergent validity of the Physical Activitiy Questionnaire for Adolescents. Ped Exerc Res 1997;9: $342-52$.

33. Voss C, Sandercock G. Aerobic fitness and mode of travel to school in English schoolchildren. Med Sci Sports Exerc 2010;42:281-7.

34. Cole TJ, Freeman JV, Preece MA. Body mass index reference curves for the UK, 1990. Arch Dis Child 1995;73:25-9.

35. Office for National Statistics (ONS). The English Indices of Deprivation 2007 (revised). London: Office of The Deputy Prime Minister, 2007.

36. Léger LA, Mercier D, Gadoury C, Lambert J. The multistage 20 metre shuttle run test for aerobic fitness. J Sports Sci 1988;6:93-101.

37. Janz KF, Lutuchy EM, Wenthe P, Levy SM. Measuring activity in children and adolescents using self-report: PAQ-C and PAQ-A. Med Sci Sports Exerc 2008; $40: 767-72$.

38. Cerin E. Ways of unraveling how and why physical activity influences mental health through statistical mediation analyses. Mental Health and Physical Activity 2010;3:51-60.

39. Cerin E, Mackinnon DP. A commentary on current practice in mediating variable analyses in behavioural nutrition and physical activity. Public Health Nutr 2009;12:1182-8.

40. Preacher KJ, Hayes AF. Asymptotic and resampling strategies for assessing and comparing indirect effects in multiple mediator models. Behav Res Methods 2008;40:879-91. 